\title{
TWO HILBERT SPACES IN WHICH POLYNOMIALS ARE NOT DENSE
}

\author{
BY \\ D. J. NEWMAN( $\left.{ }^{1}\right)$ AND D. K. WOHLGELERNTER( $\left.{ }^{2}\right)$
}

\begin{abstract}
Let $S$ be the Hilbert space of entire functions $f(z)$ such that $\|f(z)\|^{2}$ $=\iint|f(z)|^{2} d m(z)$, where $m$ is a positive measure defined on the Borel sets of the complex plane. Two Hilbert spaces are constructed in which polynomials are not dense. In the second example, our space is one which contains all exponentials and yet in which the exponentials are not complete. This is a somewhat surprising result since the exponentials are always complete on the real line.
\end{abstract}

Introduction. Let $m$ be a positive measure defined on the Borel sets of the complex plane $C$. In the quest for sufficient conditions on $m$ that ensure that the analytic polynomials be dense in the entire functions of $L^{2}(d m)$ one looks for counterexamples. A study of these counterexamples often gives some insight into the nature of the problem.

We denote by $S$ the space of entire functions $f(z)$ such that

$$
\|f(z)\|^{2}=\int|f(z)|^{2} d m(z)<\infty .
$$

The integration here is over the complex plane. $S$ is then a pre-Hilbert space where, as usual, the inner product $\langle f, g\rangle=\int f \bar{g} d m(z)$. We are interested primarily in the case where the space $S$ is a complete Hilbert space. By "dense in $S$ " we mean dense in the metric imposed by (1).

In this paper we give two examples of Hilbert spaces in which polynomials are not dense. In our first example we use the well-known fact that $\exp (i z)$ cannot be approximated by polynomials in $L^{2}[0, \infty)$ with the weight $\exp \left(-x^{\alpha}\right), 0<\alpha<\frac{1}{2}$ [1, pp. 40-45]. Hence it is really a very special case. Our second example is much more interesting in that we construct a Hilbert space containing all exponentials and yet in which the exponentials are not complete. It follows that polynomials

Received by the editors June 14, 1971 .

AMS 1970 subject classifications. Primary 30A82.

Key words and phrases. Weighted $L^{2}$-approximation, complete, dense.

${ }^{(1)}$ Research sponsored by the Air Force Office of Scientific Research, Office of Aerospace Research USAF, Grant No. AFOSR-69-1736.

$\left({ }^{2}\right)$ Research part of a doctoral dissertation written under Donald J. Newman at Yeshiva University. The author was sponsored by USAF Grant No. AFOSR-69-1736, the National Aeronautics and Space Administration Grant No. NGT-33-023-003 and the United States Steel Foundation.

Copyright $(C)$ 1972, American Mathematical Society 
are not dense. This is surprising since the exponentials are always complete on the real line.

EXAmple 1. We divide the plane into two sets $R_{1}$ and $R_{2}$ where

$$
\begin{aligned}
& R_{1}=\{z \mid \operatorname{Re} z \leqq 0 \text { or } \operatorname{Re} z>0 \text { and }|\operatorname{Im} z|>1\} \\
& R_{2}=\{z \mid \operatorname{Re} z>0 \text { and }|\operatorname{Im} z| \leqq 1\} .
\end{aligned}
$$

Let

$$
\begin{aligned}
& d m(z)=\exp \left(-|z|^{1+\delta}\right) d A_{z} \quad(\delta>0) \quad \text { for } z \text { in } R_{1} \text {, } \\
& =\exp \left(-|z|^{\alpha}\right) d A_{z} \quad\left(0<\alpha<\frac{1}{2}\right) \quad \text { for } z \text { in } R_{2} \text {. }
\end{aligned}
$$

As above, we denote by $S$ the space of entire functions $f(z)$ such that $\|f(z)\|^{2}$ $=\int|f(z)|^{2} d m(z)<\infty$. The function $e^{i z}$ is easily seen to belong to $S$. We shall show that there exists a positive constant $M$ such that for any function $f(z)$ in $S$

$$
\int_{0}^{\infty}|f(x)|^{2} \exp \left(-x^{\alpha}\right) d x<M\|f(z)\|^{2}
$$

It follows from (1.1) that in particular $e^{i z}$ cannot be approximated by polynomials in $S$. For suppose the converse, i.e. given $\varepsilon>0 \exists P(z)$ such that $\left\|e^{i z}-P(z)\right\|<\varepsilon$. But then $e^{i x}$ could be approximated on $L^{2}[0, \infty)$ with the weight $\exp \left(-x^{\alpha}\right), 0<\alpha<\frac{1}{2}$, and hence we would have a contradiction.

Let $f(z)$ be in $S$. Then for any point $u, u \geqq 0$, we have

$$
f^{2}(u)=\frac{1}{\pi} \int_{0}^{2 \pi} \int_{0}^{1} f^{2}\left(u+r e^{i \theta}\right) r d r d \theta
$$

and therefore

$$
\int_{0}^{\infty}|f(u)|^{2} \exp \left(-u^{\alpha}\right) d u \leqq \frac{1}{\pi} \int_{0}^{\infty} \exp \left(-u^{\alpha}\right) \int_{0}^{2 \pi} \int_{0}^{1}\left|f\left(u+r e^{i \theta}\right)\right|^{2} r d r d \theta d u .
$$

We let $x=u+r \cos \theta, y=r \sin u$ and $v=u$ in the right-hand integral of (1.3), and obtain

$$
\begin{aligned}
\int_{0}^{\infty} & |f(u)|^{2} \exp \left(-u^{\alpha}\right) d u \\
(1.4) & \leqq \frac{1}{\pi} \int_{-1}^{\infty} \int_{-1}^{1}|f(x+i y)|^{2} \int_{\max \{0, x-1\}}^{x+1} \exp \left(-v^{\alpha}\right) d v d y d x \\
& \leqq \frac{2}{\pi}\left[\int_{-1}^{2} \int_{-1}^{1}|f(x+i y)|^{2} d y d x+\int_{2}^{\infty} \int_{-1}^{1}|f(x+i y)|^{2} \int_{x-1}^{x+1} \exp \left(-v^{\alpha}\right) d v d y d x\right] .
\end{aligned}
$$

Let $z=x+i y$ and note that for $x \geqq 2$ and $|y|<1$ we have

(i) $v \geqq x-1=|x|+1-2 \geqq|x|+|y|-2 \geqq|z|-2$, and

(ii) $|z|^{\alpha}=(|z|-2+2)^{\alpha} \leqq(|z|-2)^{\alpha}+2^{\alpha}$. 
Combining (i) and (ii) we have $\exp \left(-v^{\alpha}\right)<\exp \left(-|z|^{\alpha}+2^{\alpha}\right)$ and hence

$$
\begin{aligned}
\int_{0}^{\infty} & |f(u)|^{2} \exp \left(-u^{\alpha}\right) d u \\
(1.5) & <M\left[\int_{-1}^{0} \int_{-1}^{1}|f(z)|^{2} \exp \left(-|z|^{1+\delta}\right) d y d x+\int_{0}^{\infty} \int_{-1}^{1}|f(z)|^{2} \exp \left(-|z|^{\alpha}\right) d y d x\right] \\
& <M\|f(z)\|^{2},
\end{aligned}
$$

which is (1.1).

REMARK. If we vary the conditions on $\operatorname{dm}(z)$ slightly, e.g., $\alpha=\frac{1}{2}$ or $\delta=0$ (in which case $e^{i z}$ does not belong to $S$ ), the situation becomes entirely different and whether polynomials are dense in the space $S$ thus defined remains an open question.

EXAMPLE 2. We now construct a Hilbert space containing all exponentials and in which the exponentials are not complete. Our construction is motivated by Example 1.

Let $z=x+i y$. Denote by $S^{\prime}$ the space of entire functions $f(z)$ such that

$$
\|f(z)\|_{S^{\prime}}^{2}=\int_{0}^{\infty}|f(x)|^{2}+|f(\xi x)|^{2} d m^{\prime}(x)<\infty
$$

where $d m^{\prime}(x)=\exp \left(-2 x^{2}\right) x^{11} /\left(1-\exp \left(-x^{8}\right)\right)$ and $\xi=\exp (i \pi / 4) . S^{\prime}$ is a preHilbert space where the inner product of two functions $f, g$ in $S^{\prime}$ is

$$
\langle f, g\rangle_{S^{\prime}}=\int_{0}^{\infty}[f(x) \bar{g}(x)+f(\xi x) \bar{g}(\xi x)] d m^{\prime}(x) .
$$

Clearly all exponentials belong to $S^{\prime}$. We exhibit a function $F(z)$ in $S^{\prime}$ which cannot be approximated by a linear combination of exponentials. We then construct a Hilbert space $S$ such that

$$
F(z) \text { belongs to } S \text {, }
$$

and

$$
\|f(z)\|_{S^{\prime}}<M\|f(z)\|_{S}
$$

where $f(z)$ is any function in $S$ and $M$ is some positive constant independent of $f(z)$. As in our first example, (2.2) implies that in particular $F(z)$ cannot be approximated by a linear combination of exponentials in $S$ and therefore the exponentials are not complete in this Hilbert space.

Let

$$
F(z)=\exp \left((1-i) z^{2}\right)\left(1-\exp \left(-z^{8}\right)\right) / z^{8} .
$$

Clearly $F(z)$ is in $S^{\prime}$ since

$$
\|F(z)\|_{S^{\prime}}^{2}=2 \int_{0}^{\infty} \frac{\left(1-\exp \left(-x^{8}\right)\right)}{x^{5}} d x<\infty .
$$


To show that $F(z)$ cannot be approximated by a linear combination of exponentials it suffices to show that

$$
\left\langle e^{\lambda z}, F(z)\right\rangle_{S^{\prime}}=0
$$

for all complex $\lambda$.

Let

$$
g(z)=z^{3} \exp \left(\lambda z-(1-i) z^{2}\right) .
$$

$g(z)$ is analytic. Moreover,

$$
\lim _{R \rightarrow \infty} \int_{0}^{\pi / 4} R\left|g\left(R e^{i \theta}\right)\right| d \theta=0
$$

Hence by contour integration we have

$$
\int_{0}^{\infty} g(x) d x-\xi \int_{0}^{\infty} g(\xi x) d x=0
$$

i.e.

$$
\int_{0}^{\infty} \exp \left(\lambda x-(1-i) x^{2}\right) x^{3} d x-\xi^{4} \int_{0}^{\infty} \exp \left(\lambda \xi x-(1+i) x^{2}\right) x^{3} d x=0
$$

$\xi^{4}=1$ and therefore (2.3) follows.

We now define our Hilbert space $S$. Let

$$
\begin{aligned}
& R_{1}=\{z|| z|>3, x>0,| x y \mid \leqq 1\}, \\
& R_{2}=\left\{z|| z|>3, x>0, y>0,| x^{2}-y^{2} \mid \leqq 2\right\}, \\
& R_{3}=\{z|| z \mid \leqq 3\}, \quad R_{4}=C-\left(R_{1} \cup R_{2} \cup R_{3}\right) .
\end{aligned}
$$

We let $d m(z)=K(z) d x d y$ where

$$
\begin{aligned}
K(z) & =\exp \left(-2 x^{2}\right) x^{12} & & \left(z \in R_{1}\right), \\
& =\exp \left(-(x+y)^{2}\right)(x+y)^{12} & & \left(z \in R_{2}\right), \\
& =1 & & \left(z \in R_{3}\right), \\
& =\exp \left(-3|z|^{8}\right) & & \left(z \in R_{4}\right) .
\end{aligned}
$$

As above $S$ is the Hilbert space of entire functions $f(z)$ such that $\int|f(z)|^{2} d m(z)<\infty$. It is obvious that the exponentials belong to $S$. Moreover one easily verifies that

$$
\begin{aligned}
\int_{R_{1}}|F(z)|^{2} d m(z) & <C \int_{2}^{\infty} \int_{-1 / x}^{1 / x} \frac{x^{12} \exp \left(-2 y^{2}+4 x y\right)}{|z|^{16}} d y d x \\
& <C \int_{2}^{\infty} \frac{1}{x^{5}} d x<\infty,
\end{aligned}
$$

where $C$ is a generic constant. 
Similarly, letting $z=\xi \eta, \eta=t+i w$ we have

$$
\begin{aligned}
\int_{R_{2}}|F(z)|^{2} d m(z) & <C \int_{2}^{\infty} \int_{-1 / t}^{1 / t} t^{12} \exp \left(-2 w^{2}-4 t w\right) d w d t \\
& <C \int_{2}^{\infty} \frac{1}{t^{5}}<\infty
\end{aligned}
$$

Obviously, $\int_{R_{3} \cup R_{4}}|F(z)|^{2} d m(z)<\infty$. We therefore conclude $\|F(z)\|_{S}<\infty$.

Finally we must prove (2.2). Let $f(z)$ be in $S$. It evidently suffices to show

$$
\|f(z)\|_{S^{\prime}}^{2}<\text { constant }\left[\int_{|z| \leqq 4}|f(z)|^{2} d A_{z}+\int_{R_{1} \cup R_{2}}|f(z)|^{2} d m(z)\right] .
$$

Indeed, for $3 \leqq|z| \leqq 4, d m(z)>m_{1}>0$. From (2.7) we shall then have

$$
\begin{aligned}
\|f(z)\|_{S^{\prime}}^{2} & <M\left[\int_{|z| \leqq 3}|f(z)|^{2} d A_{z}+\int_{3<|z| \leqq 4}|f(z)|^{2} d m(z)+\int_{R_{1} \cup R_{2}}|f(z)|^{2} d m(z)\right] \\
& <M\|f(z)\|_{S^{2}}^{2}
\end{aligned}
$$

Since $f(z)$ is entire we have for any point $u \geqq 0$

$$
f^{2}(u)=\frac{4 u^{2}}{\pi i} \int_{0}^{2 \pi} \int_{0}^{1 / 2 u} f^{2}\left(u+r e^{i \theta}\right) r d r d \theta
$$

Hence,

$$
\begin{aligned}
\int_{3}^{\infty} \frac{|f(u)|^{2} \exp \left(-2 u^{2}\right) u^{11}}{1-\exp \left(-u^{8}\right)} d u \\
\quad \leqq \frac{4 e}{\pi(e-1)} \int_{3}^{\infty} \int_{0}^{2 \pi} \int_{0}^{1 / 2 u}\left|f\left(u+r e^{i \theta}\right)\right|^{2} \exp \left(-2 u^{2}\right) u^{13} r d r d \theta d u .
\end{aligned}
$$

We let $x=u+r \cos \theta, y=r \sin \theta$ and $v=u$ in the right-hand integral of (2.9) and obtain

$$
\begin{aligned}
& \int_{3}^{\infty} \frac{|f(u)|^{2} \exp \left(-2 u^{2}\right) u^{11}}{1-\exp \left(-u^{8}\right)} d u \\
& <C\left[\int_{3-1 / 6}^{\infty} d x \int_{-1 / x}^{1 / x}|f(z)|^{2} d y \int_{x-1 / x}^{x+1 / x} \exp \left(-2 v^{2}\right) v^{13} d v\right] \\
& <C\left[\int_{3-1 / 6}^{\infty} \int_{-1 / x}^{1 / x}|f(z)|^{2} \exp \left(-2 x^{2}\right) x^{12} d y d x\right] \\
& <C\left[\int_{|z| \leqq 4}|f(z)|^{2} d A_{z}+\int_{R_{1}}|f(z)|^{2} d m(z)\right] .
\end{aligned}
$$

Similarly,

$$
\int_{0}^{3} \frac{|f(u)|^{2} \exp \left(-2 u^{2}\right)}{1-\exp \left(-u^{8}\right)} d u<C \int_{|z| \leqq 4}|f(z)|^{2} d A_{z}
$$


Let $\eta=t+i w$. In exactly the same manner we obtain

$$
\begin{aligned}
& \int_{0}^{\infty} \frac{|f(\xi u)|^{2} \exp \left(-2 u^{2}\right) u^{11}}{1-\exp \left(-u^{8}\right)} d u \\
& \quad<C\left[\int_{|\eta| \leqq 4}|f(\xi \eta)|^{2} d t d w+\int_{|\eta| \geqq 3 ;|t w| \leqq 1 ; t>0}|f(\xi \eta)|^{2} \exp \left(-2 t^{2}\right) t^{12} d t d w\right] .
\end{aligned}
$$

Letting $z=\xi \eta$ we have from (2.11)

$$
\begin{aligned}
& \int_{0}^{\infty} \frac{|f(\xi u)|^{2} \exp \left(-2 u^{2}\right)}{1-\exp \left(-u^{8}\right)} d u \\
& \quad<C\left[\int_{|z| \leqq 4}|f(z)|^{2} d x \cdot d y+\int_{R_{1}}|f(z)|^{2} \exp \left(-(x+y)^{2}\right)(x+y)^{12} d A_{z}\right] .
\end{aligned}
$$

The desired result, namely (2.7), follows from (2.10) and (2.12).

\section{REFERENCES}

1. J. Horváth, Approximation and quasi-analytic functions, Univ. Madrid Publ. Sec. Mat. Fac. Ci. I 1 (1956). (Spanish) MR 18, 389.

Department of Mathematics, Yeshiva University, New York, New York 10033

Department of Mathematics, Bernard M. Baruch College, City University of New York, New York, New York 10010 